\title{
Interactive comment on "Optimization of Sulfate Aerosol Hygroscopicity Parameter in WRF-Chem version (3.8.1)" by Ah-Hyun Kim et al.
}

Ah-Hyun Kim et al.

ssyum@yonsei.ac.kr

Received and published: 7 October 2020

See the supplement

Please also note the supplement to this comment:

https://gmd.copernicus.org/preprints/gmd-2020-170/gmd-2020-170-AC2-

supplement.pdf

Interactive comment on Geosci. Model Dev. Discuss., https://doi.org/10.5194/gmd-2020-170, 2020. 